\title{
NEW GENERALIZED MIDPOINT TYPE INEQUALITIES FOR FRACTIONAL INTEGRAL
}

\author{
H. BUDAK AND P. AGARWAL
}

Received 2 June, 2019

\begin{abstract}
Here, our first aim to establish a new identity for differentiable function involving Riemann-Liouville fractional integrals. Then, we obtain same generalized midpoint type inequalities utilizing convex and concave function.
\end{abstract}

2010 Mathematics Subject Classification: 26D15; 26B25; 26D10

Keywords: Hermite-Hadamard inequality, fractional integral operators, convex function, concave function

\section{INTRODUCTION}

In recent years, the Hermite-Hadamard inequality, which is the first fundamental result for convex mappings with a natural geometrical interpretation and many applications, has drawn attention much interest in elementary mathematics.

The inequalities discovered by C. Hermite and J. Hadamard for convex functions are considerable significant in the literature (see, e.g.,[17, p.137], [7]). These inequalities state that if $f: I \rightarrow \mathbb{R}$ is a convex function on the interval $I$ of real numbers and $a, b \in I$ with $a<b$, then

$$
f\left(\frac{a+b}{2}\right) \leq \frac{1}{b-a} \int_{a}^{b} f(x) d x \leq \frac{f(a)+f(b)}{2} .
$$

Both inequalities hold in the reversed direction if $f$ is concave.

In [12], U. S. Kurmac1 give the following identity and using this identiy, obtain some bounds for the left hand side of the inequality (1.1)

Lemma 1. Let $f: I^{*} \rightarrow \mathbb{R}$ be differentiable function on $I^{*}, a, b \in I^{*}\left(I^{*}\right.$ is interior of I) with $a<b$. If $f^{\prime} \in L[a, b]$, then we have

$$
\frac{1}{b-a} \int_{a}^{b} f(t) d t-f\left(\frac{a+b}{2}\right)
$$




$$
=(b-a)\left[\int_{0}^{\frac{1}{2}} t f^{\prime}(t a+(1-t) b) d t+\int_{\frac{1}{2}}^{1}(1-t) f^{\prime}(t a+(1-t) b) d t\right] .
$$

Over the last twenty years, the numerous studies have focused on to obtain new bound for left hand side and right and side of the inequality (1.1). For some examples, please refer to $([2,4,6-8,14,15,18,19,24])$

In the following we will give some necessary definitions and mathematical preliminaries of fractional calculus theory which are used further in this paper.

Definition 1. Let $f \in L_{1}[a, b]$. The Riemann-Liouville integrals $J_{a+}^{\alpha} f$ and $J_{b-}^{\alpha} f$ of order $\alpha>0$ with $a \geq 0$ are defined by

$$
J_{a+}^{\alpha} f(x)=\frac{1}{\Gamma(\alpha)} \int_{a}^{x}(x-t)^{\alpha-1} f(t) d t, x>a
$$

and

$$
J_{b-}^{\alpha} f(x)=\frac{1}{\Gamma(\alpha)} \int_{x}^{b}(t-x)^{\alpha-1} f(t) d t, \quad x<b
$$

respectively. Here, $\Gamma(\alpha)$ is the Gamma function and $J_{a+}^{0} f(x)=J_{b-}^{0} f(x)=f(x)$.

It is remarkable that Sarikaya et al.[22] first give the following interesting integral inequalities of Hermite-Hadamard type involving Riemann-Liouville fractional integrals.

Theorem 1. Let $f:[a, b] \rightarrow \mathbb{R}$ be a positive function with $0 \leq a<b$ and $f \in$ $L_{1}[a, b]$. If $f$ is a convex function on $[a, b]$, then the following inequalities for fractional integrals hold:

$$
f\left(\frac{a+b}{2}\right) \leq \frac{\Gamma(\alpha+1)}{2(b-a)^{\alpha}}\left[J_{a+}^{\alpha} f(b)+J_{b-}^{\alpha} f(a)\right] \leq \frac{f(a)+f(b)}{2}
$$

with $\alpha>0$.

Sarıkaya and Yıldırım also give the following Hermite-Hadamard type inequality for the Riemann-Lioville fractional integrals in [23].

Theorem 2. Let $f:[a, b] \rightarrow \mathbb{R}$ be a positive function with $a<b$ and $f \in L_{1}[a, b]$. If $f$ is a convex function on $[a, b]$, then the following inequalities for fractional integrals hold:

$$
f\left(\frac{a+b}{2}\right) \leq \frac{2^{\alpha-1} \Gamma(\alpha+1)}{(b-a)^{\alpha}}\left[J_{\left(\frac{a+b}{2}\right)}^{\alpha} f(b)+J_{\left(\frac{a+b}{2}\right)^{\alpha}}^{-f(a)}\right] \leq \frac{f(a)+f(b)}{2} .
$$


Whereupon Sarikaya et al. obtain the Hermite-Hadamard inequality for RiemannLioville fractional integrals, many authors have studied to generalize this inequality and establish Hermite-Hadamard inequality other fractional integrals such as $k$ fractional integral, Hadamard fractional integrals, Katugampola frtactional integrals, Conformable fractional integrals, etc. For some of them, please see $([1,3,5,9,11,13$, 16,20,21,25-27]). For more information about fraction calculus please refer to [10].

In the following section, we establish some new generalized midpoint type inequalities for Riemann-Liouville fractional integrals.

\section{Generalized MIDPoint INEQUALITIES FOR RiEMANN-LiOUVILLE FRACTIONAL INTEGRAL OPERATORS}

First, we give the following lemma which will be used frequently later.

Lemma 2. Let $f:[a, b] \rightarrow \mathbb{R}$ be a differentiable mapping on $(a, b)$ with $a<b$. If $f^{\prime} \in L[a, b]$, then for all $x \in[a, b]$ the following equality for fractional integrals holds:

$$
\begin{aligned}
& \frac{\Gamma(\alpha+1)}{b-a}\left[(x-a)^{1-\alpha} J_{b-}^{\alpha} f(a+b-x)+(b-x)^{1-\alpha} J_{a+}^{\alpha} f(a+b-x)\right] \\
& -f(a+b-x) \\
= & \frac{(x-a)^{2}}{b-a} \int_{0}^{1}\left(1-t^{\alpha}\right) f^{\prime}(t b+(1-t)(a+b-x)) d t \\
& +\frac{(b-x)^{2}}{b-a} \int_{0}^{1}\left(t^{\alpha}-1\right) f^{\prime}(t a+(1-t)(a+b-x)) d t .
\end{aligned}
$$

Proof. Integrating the by parts, we have

$$
\begin{aligned}
& \int_{0}^{1}\left(1-t^{\alpha}\right) f^{\prime}(t b+(1-t)(a+b-x)) d t \\
= & \left.\left(1-t^{\alpha}\right) \frac{f^{\prime}(t b+(1-t)(a+b-x))}{x-a}\right|_{0} ^{1} \\
& +\frac{\alpha}{x-a} \int_{0}^{1} t^{\alpha-1} f^{\prime}(t b+(1-t)(a+b-x)) d t
\end{aligned}
$$




$$
\begin{aligned}
& =-\frac{f(a+b-x)}{x-a}+\frac{\Gamma(1+\alpha)}{(x-a)^{\alpha+1}} \int_{a+b-x}^{b}(u-(a+b-x))^{\alpha-1} f(u) d u \\
& =-\frac{f(a+b-x)}{x-a}+\frac{\Gamma(1+\alpha)}{(x-a)^{\alpha+1}} J_{b-}^{\alpha} f(a+b-x) .
\end{aligned}
$$

Similarly, we get

$$
\begin{aligned}
& \int_{0}^{1}\left(t^{\alpha}-1\right) f^{\prime}(t a+(1-t)(a+b-x)) d t \\
= & -\frac{f(a+b-x)}{b-x}+\frac{\Gamma(1+\alpha)}{(b-x)^{\alpha+1}} J_{a+}^{\alpha} f(a+b-x) .
\end{aligned}
$$

By the identities (2.2) and (2.3), we obtain the required result (2.1).

Theorem 3. $f:[a, b] \rightarrow \mathbb{R}$ be a differentiable mapping on $(a, b)$ with $0 \leq a<b$. If $\left|f^{\prime}\right|^{q}$ is convex on $[a, b]$ for some fixed $q>1$, then for all $x \in[a, b]$ following inequality for fractional integrals holds:

$$
\begin{aligned}
& \quad \frac{\Gamma(\alpha+1)}{b-a}\left[(x-a)^{1-\alpha} J_{b-}^{\alpha} f(a+b-x)+(b-x)^{1-\alpha} J_{a+}^{\alpha} f(a+b-x)\right] \\
& -f(a+b-x) \mid \\
& \leq \frac{1}{b-a}\left(\frac{\alpha p}{\alpha p+1}\right)^{\frac{1}{p}}\left[(x-a)^{2}\left[\frac{\left|f^{\prime}(b)\right|^{q}+\left|f^{\prime}(a+b-x)\right|^{q}}{2}\right]^{\frac{1}{q}}\right. \\
& \left.\quad+(b-x)^{2}\left[\frac{\left|f^{\prime}(a)\right|^{q}+\left|f^{\prime}(a+b-x)\right|^{q}}{2}\right]^{\frac{1}{q}}\right]
\end{aligned}
$$

where $\frac{1}{p}+\frac{1}{q}=1$.

Proof. By the Lemma 2, we have

$$
\begin{aligned}
& \mid \frac{\Gamma(\alpha+1)}{b-a}\left[(x-a)^{1-\alpha} J_{b-}^{\alpha} f(a+b-x)+(b-x)^{1-\alpha} J_{a+}^{\alpha} f(a+b-x)\right] \\
& -f(a+b-x) \mid \\
\leq & \frac{(x-a)^{2}}{b-a} \int_{0}^{1}\left|1-t^{\alpha}\right|\left|f^{\prime}(t b+(1-t)(a+b-x))\right| d t
\end{aligned}
$$




$$
+\frac{(b-x)^{2}}{b-a} \int_{0}^{1}\left|t^{\alpha}-1\right|\left|f^{\prime}(t a+(1-t)(a+b-x))\right| d t .
$$

Using the Hölder's inequality and convexity of $\left|f^{\prime}\right|^{q}$, we obtain

$$
\begin{aligned}
& \int_{0}^{1}\left|1-t^{\alpha}\right|\left|f^{\prime}(t b+(1-t)(a+b-x))\right| d t \\
\leq & \left(\int_{0}^{1}\left|1-t^{\alpha}\right|^{p} d t\right)^{\frac{1}{p}}\left(\int_{0}^{1}\left|f^{\prime}(t b+(1-t)(a+b-x))\right|^{q} d t\right)^{\frac{1}{q}} \\
\leq & \left(\int_{0}^{1}\left(1-t^{p \alpha}\right) d t\right)^{\frac{1}{p}}\left(\int_{0}^{1}\left[t\left|f^{\prime}(b)\right|^{q}+(1-t)\left|f^{\prime}(a+b-x)\right|^{q}\right] d t\right)^{\frac{1}{q}} \\
= & \left(\frac{\alpha p}{\alpha p+1}\right)^{\frac{1}{p}}\left(\frac{\left|f^{\prime}(b)\right|^{q}+\left|f^{\prime}(a+b-x)\right|^{q}}{2}\right)^{\frac{1}{q}} .
\end{aligned}
$$

Here we use

$$
(A-B)^{q} \leq A^{q}-B^{q},
$$

for any $A>B \geq 0$ and $q \geq 1$.

Similarly we have

$$
\begin{aligned}
& \int_{0}^{1}\left|t^{\alpha}-1\right|\left|f^{\prime}(t a+(1-t)(a+b-x))\right| d t \\
\leq & \left(\frac{\alpha p}{\alpha p+1}\right)^{\frac{1}{p}}\left(\frac{\left|f^{\prime}(a)\right|^{q}+\left|f^{\prime}(a+b-x)\right|^{q}}{2}\right)^{\frac{1}{q}} .
\end{aligned}
$$

If we substitute the inequalities (2.6) and (2.7) in (2.5), then we obtain the desired result.

Corollary 1. Under assumption of Theorem 3 with $x=\frac{a+b}{2}$, we have the following inequality

$$
\begin{aligned}
& \left|\frac{2^{\alpha-1} \Gamma(\alpha+1)}{(b-a)^{\alpha}}\left[J_{b-}^{\alpha} f\left(\frac{a+b}{2}\right)+J_{a+}^{\alpha} f\left(\frac{a+b}{2}\right)\right]-f\left(\frac{a+b}{2}\right)\right| \\
\leq & \frac{b-a}{4}\left(\frac{\alpha p}{\alpha p+1}\right)^{\frac{1}{p}}\left[\left(\frac{3\left|f^{\prime}(a)\right|^{q}+\left|f^{\prime}(b)\right|^{q}}{4}\right)^{\frac{1}{q}}+\left(\frac{3\left|f^{\prime}(b)\right|^{q}+\left|f^{\prime}(a)\right|^{q}}{4}\right)^{\frac{1}{q}}\right]
\end{aligned}
$$


$\leq \frac{b-a}{4}\left(\frac{4 \alpha p}{\alpha p+1}\right)^{\frac{1}{p}}\left[\left|f^{\prime}(a)\right|+\left|f^{\prime}(b)\right|\right]$.

Proof. The proof of the first inequality in (2.8) is obvious from the convexity of $\left|f^{\prime}\right|^{q}$. For the proof of second inequality, let $a_{1}=3\left|f^{\prime}(a)\right|^{q}, b_{1}=\left|f^{\prime}(b)\right|^{q}, a_{2}=$ $\left|f^{\prime}(a)\right|^{q}$ and $b_{2}=3\left|f^{\prime}(b)\right|^{q}$. Using the fact that,

$$
\sum_{k=1}^{n}\left(a_{k}+b_{k}\right)^{s} \leq \sum_{k=1}^{n} a_{k}^{s}+\sum_{k=1}^{n} b_{k}^{s}, 0 \leq s<1
$$

the desired result can be obtained straightforwardly.

Theorem 4. $f:[a, b] \rightarrow \mathbb{R}$ be a differentiable mapping on $(a, b)$ with $0 \leq a<b$. If $\left|f^{\prime}\right|^{q}$ is convex on $[a, b]$ for some fixed $q \geq 1$, then for all $x \in[a, b]$ following inequality for fractional integrals holds:

$$
\begin{aligned}
& \quad \mid \frac{\Gamma(\alpha+1)}{b-a}\left[(x-a)^{1-\alpha} J_{b-}^{\alpha} f(a+b-x)+(b-x)^{1-\alpha} J_{a+}^{\alpha} f(a+b-x)\right] \\
& -f(a+b-x) \mid \\
& \leq \frac{1}{b-a}\left(\frac{\alpha}{\alpha+1}\right)^{1-\frac{1}{q}} \\
& \quad \times\left[(x-a)^{2}\left(\frac{\alpha}{2(\alpha+2)}\left|f^{\prime}(b)\right|^{q}+\left[\frac{(\alpha+1)(\alpha+2)-2}{2(\alpha+1)(\alpha+2)}\right]\left|f^{\prime}(a+b-x)\right|^{q}\right)^{\frac{1}{q}}\right. \\
& \left.\quad+(b-x)^{2}\left(\frac{\alpha}{2(\alpha+2)}\left|f^{\prime}(a)\right|^{q}+\left[\frac{(\alpha+1)(\alpha+2)-2}{2(\alpha+1)(\alpha+2)}\right]\left|f^{\prime}(a+b-x)\right|^{q}\right)^{\frac{1}{q}}\right] .
\end{aligned}
$$

Proof. By the Lemma 2 and the power mean inequality, we have

$$
\begin{aligned}
& \mid \frac{\Gamma(\alpha+1)}{b-a}\left[(x-a)^{1-\alpha} J_{b-}^{\alpha} f(a+b-x)+(b-x)^{1-\alpha} J_{a+}^{\alpha} f(a+b-x)\right] \\
& -f(a+b-x) \mid \\
\leq & \frac{(x-a)^{2}}{b-a} \int_{0}^{1}\left|1-t^{\alpha}\right|\left|f^{\prime}(t b+(1-t)(a+b-x))\right| d t \\
& +\frac{(b-x)^{2}}{b-a} \int_{0}^{1}\left|t^{\alpha}-1\right|\left|f^{\prime}(t a+(1-t)(a+b-x))\right| d t .
\end{aligned}
$$




$$
\begin{aligned}
\leq & \frac{(x-a)^{2}}{b-a}\left(\int_{0}^{1}\left|1-t^{\alpha}\right| d t\right)^{1-\frac{1}{q}} \\
& \times\left(\int_{0}^{1}\left|1-t^{\alpha}\right|\left|f^{\prime}(t b+(1-t)(a+b-x))\right|^{q} d t\right)^{\frac{1}{q}} \\
& +\frac{(b-x)^{2}}{b-a}\left(\int_{0}^{1}\left|t^{\alpha}-1\right| d t\right)^{1-\frac{1}{q}} \\
& \times\left(\int_{0}^{1}\left|t^{\alpha}-1\right|\left|f^{\prime}(t a+(1-t)(a+b-x))\right|^{q} d t\right)^{\frac{1}{q}} .
\end{aligned}
$$

Using the convexity of $\left|f^{\prime}\right|^{q}$, we obtain

$$
\begin{aligned}
& \int_{0}^{1}\left|1-t^{\alpha}\right|\left|f^{\prime}(t b+(1-t)(a+b-x))\right|^{q} d t \\
\leq & \int_{0}^{1}\left(1-t^{\alpha}\right)\left[t\left|f^{\prime}(b)\right|^{q}+(1-t)\left|f^{\prime}(a+b-x)\right|^{q}\right] d t \\
= & \frac{\alpha}{2(\alpha+2)}\left|f^{\prime}(b)\right|^{q}+\left[\frac{(\alpha+1)(\alpha+2)-2}{2(\alpha+1)(\alpha+2)}\right]\left|f^{\prime}(a+b-x)\right|^{q}
\end{aligned}
$$

and similarly we have

$$
\begin{aligned}
& \int_{0}^{1}\left|t^{\alpha}-1\right|\left|f^{\prime}(t a+(1-t)(a+b-x))\right|^{q} d t \\
\leq & \frac{\alpha}{2(\alpha+2)}\left|f^{\prime}(a)\right|^{q}+\left[\frac{(\alpha+1)(\alpha+2)-2}{2(\alpha+1)(\alpha+2)}\right]\left|f^{\prime}(a+b-x)\right|^{q}
\end{aligned}
$$

which completes the proof.

Corollary 2. Under assumption of Theorem 4 with $x=\frac{a+b}{2}$, we have the following inequality

$$
\left|\frac{2^{\alpha-1} \Gamma(\alpha+1)}{(b-a)^{\alpha}}\left[J_{b-}^{\alpha} f\left(\frac{a+b}{2}\right)+J_{a+}^{\alpha} f\left(\frac{a+b}{2}\right)\right]-f\left(\frac{a+b}{2}\right)\right|
$$




$$
\begin{aligned}
\leq & \frac{b-a}{4}\left(\frac{\alpha}{\alpha+1}\right)^{1-\frac{1}{q}} \\
& \times\left[\left(\frac{\alpha(3 \alpha+5)}{4(\alpha+1)(\alpha+2)}\left|f^{\prime}(b)\right|^{q}+\left[\frac{(\alpha+1)(\alpha+2)-2}{4(\alpha+1)(\alpha+2)}\right]\left|f^{\prime}(a)\right|^{q}\right)^{\frac{1}{q}}\right. \\
& \left.+\left(\frac{\alpha(3 \alpha+5)}{4(\alpha+1)(\alpha+2)}\left|f^{\prime}(a)\right|^{q}+\left[\frac{(\alpha+1)(\alpha+2)-2}{4(\alpha+1)(\alpha+2)}\right]\left|f^{\prime}(b)\right|^{q}\right)^{\frac{1}{q}}\right] .
\end{aligned}
$$

Theorem 5. $f:[a, b] \rightarrow \mathbb{R}$ be a differentiable mapping on $(a, b)$ with $0 \leq a<b$. If $\left|f^{\prime}\right|^{q}$ is concave on $[a, b]$ for some fixed $q>1$, then for all $x \in[a, b]$ following inequality for fractional integrals holds:

$$
\begin{aligned}
& \mid \frac{\Gamma(\alpha+1)}{b-a}\left[(x-a)^{1-\alpha} J_{b-}^{\alpha} f(a+b-x)+(b-x)^{1-\alpha} J_{a+}^{\alpha} f(a+b-x)\right] \\
& -f(a+b-x) \mid \\
& \leq \frac{1}{b-a}\left(\frac{\alpha p}{\alpha p+1}\right)^{\frac{1}{p}}\left[(x-a)^{2}\left|f^{\prime}\left(\frac{a+2 b-x}{2}\right)\right|+(b-x)^{2}\left|f^{\prime}\left(\frac{2 a+b-x}{2}\right)\right|\right]
\end{aligned}
$$

where $\frac{1}{q}+\frac{1}{p}=1$.

Proof. By the Lemma 2 and the Hölder inequality, we have

$$
\begin{aligned}
& \mid \frac{\Gamma(\alpha+1)}{b-a}\left[(x-a)^{1-\alpha} J_{b-}^{\alpha} f(a+b-x)+(b-x)^{1-\alpha} J_{a+}^{\alpha} f(a+b-x)\right] \\
& -f(a+b-x) \mid \\
\leq & \frac{(x-a)^{2}}{b-a} \int_{0}^{1}\left|1-t^{\alpha}\right|\left|f^{\prime}(t b+(1-t)(a+b-x))\right| d t \\
& +\frac{(b-x)^{2}}{b-a} \int_{0}^{1}\left|t^{\alpha}-1\right|\left|f^{\prime}(t a+(1-t)(a+b-x))\right| d t \\
\leq & \frac{(x-a)^{2}}{b-a}\left(\int_{0}^{1}\left(1-t^{\alpha}\right)^{p} d t\right)^{\frac{1}{p}}\left(\int_{0}^{1}\left|f^{\prime}(t b+(1-t)(a+b-x))\right|^{q} d t\right)^{\frac{1}{q}}
\end{aligned}
$$




$$
+\frac{(b-x)^{2}}{b-a}\left(\int_{0}^{1}\left(1-t^{\alpha}\right)^{p} d t\right)^{\frac{1}{p}}\left(\int_{0}^{1}\left|f^{\prime}(t a+(1-t)(a+b-x))\right|^{q} d t\right)^{\frac{1}{q}} .
$$

Since $\left|f^{\prime}\right|^{q}$ is concave on $[a, b]$, by using Jensen integral inequality, we obtain

$$
\begin{aligned}
& \int_{0}^{1}\left|f^{\prime}(t b+(1-t)(a+b-x))\right|^{q} d t \\
= & \int_{0}^{1} t^{0}\left|f^{\prime}(t b+(1-t)(a+b-x))\right|^{q} d t \\
\leq & \left.\left(\int_{0}^{1} t^{0} d t\right)\left|f^{\prime}\left(\frac{1}{\int_{0}^{1} t^{0} d t} \int_{0}^{1} t^{0}(t b+(1-t)(a+b-x)) d t\right)\right|\right|^{q} \\
= & \left|f^{\prime}\left(\frac{a+2 b-x}{2}\right)\right|^{q}
\end{aligned}
$$

and similarly,

$$
\int_{0}^{1}\left|f^{\prime}(t a+(1-t)(a+b-x))\right|^{q} d t \leq\left|f^{\prime}\left(\frac{2 a+b-x}{2}\right)\right|^{q} .
$$

By substituting the inequalities (2.11) and (2.12) in (2.10) and using the fact that

$$
\int_{0}^{1}\left(1-t^{\alpha}\right)^{p} d t \leq \int_{0}^{1}\left(1-t^{p \alpha}\right) d t=\frac{\alpha p}{\alpha p+1}
$$

we obtain the desired result.

Corollary 3. Under assumptions of Theorem 5, if we choose $x=\frac{a+b}{2}$, then we have the inequality

$$
\begin{aligned}
& \left|\frac{2^{\alpha-1} \Gamma(\alpha+1)}{(b-a)^{\alpha}}\left[J_{b-}^{\alpha} f\left(\frac{a+b}{2}\right)+J_{a+}^{\alpha} f\left(\frac{a+b}{2}\right)\right]-f\left(\frac{a+b}{2}\right)\right| \\
\leq & \left(\frac{\alpha p}{\alpha p+1}\right)^{\frac{1}{p}}\left(\frac{b-a}{4}\right)\left[\left|f^{\prime}\left(\frac{a+3 b}{4}\right)\right|+\left|f^{\prime}\left(\frac{3 a+b}{4}\right)\right|\right] .
\end{aligned}
$$


Theorem 6. $f:[a, b] \rightarrow \mathbb{R}$ be a differentiable mapping on $(a, b)$ with $0 \leq a<b$. If $\left|f^{\prime}\right|^{q}$ is concave on $[a, b]$ for some fixed $q \geq 1$, then for all $x \in[a, b]$ following inequality for fractional integrals holds:

$$
\begin{aligned}
& \mid \frac{\Gamma(\alpha+1)}{b-a}\left[(x-a)^{1-\alpha} J_{b-}^{\alpha} f(a+b-x)+(b-x)^{1-\alpha} J_{a+}^{\alpha} f(a+b-x)\right] \\
& -f(a+b-x) \mid \\
\leq & \frac{1}{b-a}\left(\frac{\alpha}{\alpha+1}\right)^{2-\frac{1}{q}}\left[(x-a)^{2}\left|f^{\prime}\left(\frac{2 \alpha(\alpha+2) b+[(\alpha+1)(\alpha+2)-2](a-x)}{2(\alpha+2)}\right)\right|^{q}\right. \\
& \left.+(b-x)^{2}\left|f^{\prime}\left(\frac{2 \alpha(\alpha+2) a+[(\alpha+1)(\alpha+2)-2](b-x)}{2(\alpha+2)}\right)\right|^{q}\right] .
\end{aligned}
$$

Proof. From the ineqauality (2.9) we have

$$
\begin{aligned}
& \frac{\Gamma(\alpha+1)}{b-a}\left[(x-a)^{1-\alpha} J_{b-}^{\alpha} f(a+b-x)+(b-x)^{1-\alpha} J_{a+}^{\alpha} f(a+b-x)\right] \\
& -f(a+b-x) \mid \\
\leq & \frac{(x-a)^{2}}{b-a}\left(\int_{0}^{1}\left|1-t^{\alpha}\right| d t\right)^{1-\frac{1}{q}}\left(\int_{0}^{1}\left|1-t^{\alpha}\right|\left|f^{\prime}(t b+(1-t)(a+b-x))\right|^{q} d t\right)^{\frac{1}{q}} \\
& +\frac{(b-x)^{2}}{b-a}\left(\int_{0}^{1}\left|t^{\alpha}-1\right| d t\right)^{1-\frac{1}{q}}\left(\int_{0}^{1}\left|t^{\alpha}-1\right|\left|f^{\prime}(t a+(1-t)(a+b-x))\right|^{q} d t\right)^{\frac{1}{q}} .
\end{aligned}
$$

Since $\left|f^{\prime}\right|^{q}$ is concave on $[a, b]$, by using Jensen integral inequality, we obtain

$$
\begin{aligned}
& \int_{0}^{1}\left|1-t^{\alpha}\right|\left|f^{\prime}(t b+(1-t)(a+b-x))\right|^{q} d t \\
\leq & \left(\int_{0}^{1}\left(1-t^{\alpha}\right) d t\right) f^{\prime}\left(\frac{1}{\int_{0}^{1}\left(1-t^{\alpha}\right) d t} \int_{0}^{1}\left(1-t^{\alpha}\right)(t b+(1-t)(a+b-x)) d t\right) \\
= & \frac{\alpha}{\alpha+1}\left|f^{\prime}\left(\frac{2 \alpha(\alpha+2) b+[(\alpha+1)(\alpha+2)-2](a-x)}{2(\alpha+2)}\right)\right|^{q}
\end{aligned}
$$

and similarly,

$$
\int_{0}^{1}\left|t^{\alpha}-1\right|\left|f^{\prime}(t a+(1-t)(a+b-x))\right|^{q} d t
$$




$$
\leq \frac{\alpha}{\alpha+1}\left|f^{\prime}\left(\frac{2 \alpha(\alpha+2) a+[(\alpha+1)(\alpha+2)-2](b-x)}{2(\alpha+2)}\right)\right|^{q} .
$$

This completes the proof.

Corollary 4. Under assumptions of Theorem 5 with $x=\frac{a+b}{2}$, then we have the inequality

$$
\begin{aligned}
& \left|\frac{2^{\alpha-1} \Gamma(\alpha+1)}{(b-a)^{\alpha}}\left[J_{b-}^{\alpha} f\left(\frac{a+b}{2}\right)+J_{a+}^{\alpha} f\left(\frac{a+b}{2}\right)\right]-f\left(\frac{a+b}{2}\right)\right| \\
\leq & \left(\frac{\alpha}{\alpha+1}\right)^{2-\frac{1}{q}}\left(\frac{b-a}{4}\right)\left[\left|f^{\prime}\left(\frac{\alpha(3 \alpha+5) b+[(\alpha+1)(\alpha+2)-2] a}{4(\alpha+2)}\right)\right|^{q}\right. \\
& \left.+\left|f^{\prime}\left(\frac{\alpha(3 \alpha+5) a+[(\alpha+1)(\alpha+2)-2] b}{4(\alpha+2)}\right)\right|^{q}\right] .
\end{aligned}
$$

\section{REFERENCES}

[1] B. Ahmad, A. Alsaedi, M. Kirane, and B. T. Torebek, "Hermite-hadamard, hermite-hadamardfejér, dragomir-agarwal and pachpatte type inequalities for convex functions via new fractional integrals," Journal of Computational and Applied Mathematics, vol. 353, pp. 120-129, 2019, doi: 10.1016/j.cam.2018.12.030.

[2] M. Alomari, M. Darus, and U. S. Kirmaci, "Refinements of hadamard-type inequalities for quasi-convex functions with applications to trapezoidal formula and to special means," Computers \& mathematics with applications, vol. 59, no. 1, pp. 225-232, 2010, doi: 10.1016/j.camwa.2009.08.002.

[3] G. Anastassiou, "General fractional hermite-hadamard inequalities using m-convexity and (s, m)convexity," Frontiers in Time Scales and Inequalities, vol. 237, p. 255, 2016.

[4] A. G. Azpeitia, "Convex functions and the hadamard inequality," Rev. Colombiana Mat, vol. 28, no. 1, pp. 7-12, 1994.

[5] H. Chen and U. N. Katugampola, "Hermite-hadamard and hermite-hadamard-fejér type inequalities for generalized fractional integrals," Journal of Mathematical Analysis and Applications, vol. 446, no. 2, pp. 1274-1291, 2017, doi: 10.1016/j.jmaa.2016.09.018.

[6] J. de la Cal, J. Cárcamo, and L. Escauriaza, "A general multidimensional hermite-hadamard type inequality," Journal of Mathematical Analysis and Applications, vol. 356, no. 2, pp. 659-663, 2009, doi: 10.1016/j.jmaa.2009.03.044.

[7] S. S. Dragomir and C. Pearce, "Selected topics on hermite-hadamard inequalities and applications," Mathematics Preprint Archive, vol. 2003, no. 3, pp. 463-817, 2003.

[8] S. Dragomir and R. Agarwal, "Two inequalities for differentiable mappings and applications to special means of real numbers and to trapezoidal formula," Applied Mathematics Letters, vol. 11, no. 5, pp. 91-95, 1998, doi: 10.1016/S0893-9659(98)00086-X.

[9] G. Farid, A. U. Rehman, and M. Zahra, "On hadamard-type inequalities for $k$-fractional integrals," Konuralp Journal of Mathematics, vol. 4, no. 2, pp. 79-86, 2016.

[10] R. Gorenflo and F. Mainardi, Fractional calculus: integral and differential equations of fractional order. New York: Springer, 1997, vol. 378.

[11] M. Iqbal, S. Qaisar, and M. Muddassar, "A short note on integral inequality of type hermitehadamard through convexity," J. Comput. Anal. Appl, vol. 21, no. 5, pp. 946-953, 2016. 
[12] U. S. Kirmaci, "Inequalities for differentiable mappings and applications to special means of real numbers and to midpoint formula," Applied Mathematics and Computation, vol. 147, no. 1, pp. 137-146, 2004, doi: 10.1016/S0096-3003(02)00657-4.

[13] M. A. Noor and M. U. Awan, "Some integral inequalities for two kinds of convexities via fractional integrals," Trans. J. Math. Mech, vol. 5, no. 2, pp. 129-136, 2013.

[14] M. E. Özdemir, M. Avci, and H. Kavurmaci, "Hermite-hadamard-type inequalities via $(\alpha, \mathrm{m})$ convexity," Computers \& Mathematics with Applications, vol. 61, no. 9, pp. 2614-2620, 2011, doi: 10.1016/j.camwa.2011.02.053.

[15] M. E. Özdemir, M. Avc1, and E. Set, "On some inequalities of hermite-hadamard type via mconvexity," Applied Mathematics Letters, vol. 23, no. 9, pp. 1065-1070, 2010.

[16] M. E. Özdemir, M. Avci-Ardiç, and H. Kavurmaci-Önalan, "Hermite-hadamard type inequalities for s-convex and s-concave functions via fractional integrals," Turkish Journal of Science, vol. 1, no. 1 , pp. 28-40, 2016.

[17] J. E. Pecaric and Y. L. Tong, Convex functions, partial orderings, and statistical applications. Academic Press, 1992.

[18] S. Qaisar and S. Hussain, "On hermite-hadamard type inequalities for functions whose first derivative absolute values are convex and concave," Fasciculi Mathematici, vol. 58, no. 1, pp. 155-166, 2017.

[19] A. Saglam, M. Z. Sarikaya, and H. Yildirim, "Some new inequalities of hermite-hadamard's type," Kyungpook Mathematical Journal, vol. 50, pp. 399-410, 2010.

[20] M. Z. Sarıkaya, A. Akkurt, H. Budak, M. E. Yıldırım, and H. Yıldırım, "Hermite-hadamard's inequalities for conformable fractional integrals," An International Journal of Optimization and Control: Theories \& Applications (IJOCTA), vol. 9, no. 1, pp. 49-59, 2019, doi: 10.11121/ijocta.01.2019.00559.

[21] M. Z. Sarikaya and H. Budak, "Generalized hermite-hadamard type integral inequalities for fractional integrals," Filomat, vol. 30, no. 5, pp. 1315-1326, 2016, doi: 10.2298/FIL1605315S.

[22] M. Z. Sarikaya, E. Set, H. Yaldiz, and N. Başak, "Hermite-hadamard's inequalities for fractional integrals and related fractional inequalities," Mathematical and Computer Modelling, vol. 57, no. 9-10, pp. 2403-2407, 2013, doi: 10.1016/j.mcm.2011.12.048.

[23] M. Z. Sarikaya and H. Yildirim, "On hermite-hadamard type inequalities for riemann-liouville fractional integrals," Miskolc Mathematical Notes, vol. 17, no. 2, pp. 1049-1059, 2016.

[24] E. Set, M. E. Ozdemir, and M. Z. Sarikaya, "New inequalities of ostrowski's type for s-convex functions in the second sense with applications," Facta Universitatis, Ser. Math. Inform, vol. 27, no. 1, pp. 67-82, 2012.

[25] E. Set, M. Z. Sarikaya, M. E. Özdemir, and H. Yıldırım, "The hermite-hadamard's inequality for some convex functions via fractional integrals and related results," Journal of Applied Mathematics, Statistics and Informatics, vol. 10, no. 2, pp. 69-83, 2014.

[26] J. Wang, X. Li, C. Zhu et al., "Refinements of hermite-hadamard type inequalities involving fractional integrals," Bulletin of the Belgian Mathematical Society-Simon Stevin, vol. 20, no. 4, pp. 655-666, 2013.

[27] Y. Zhang and J. Wang, "On some new hermite-hadamard inequalities involving riemann-liouville fractional integrals," Journal of Inequalities and Applications, vol. 2013, no. 1, p. 220, 2013, doi: 10.1186/1029-242X-2013-220.

Authors' addresses

\section{H. Budak}

Department of Mathematics, Faculty of Science and Arts, Düzce University, Düzce,Turkey

E-mail address: hsyn.budakegmail.com 


\section{P. Agarwal}

Department of Mathematics, Anand International College of Engineering, Near Kanota, Agra Road, Jaipur-303012, Rajasthan, INDIA

E-mail address: goyal.praveen2011@gmail.com 